\title{
3D Printed Masks and Transfer Stamping Process to Enable the Fabrication of the Hemispherical Organic Photodiodes
}

\author{
Hyunsoo Kim, Joosung Moon, Kyusang Lee, and Jerzy Kanicki*
}

This paper demonstrates the methods that allow the fabrication of the hemispherical organic photodiode arrays on curved concave glass substrate. They include a novel 3D shadow mask, fabricated by additive manufacturing process (stereolithography), used for the electrode deposition and 3D shaped polydimethylsiloxane stamp used for organic active layer formation. These techniques allow the direct patterning of the organic photodiode devices on hemispherical substrates without an excessive strain or deformation. It is believed that the developed 3D mask and 3D organic layer transfer printing process can be further utilized for fabrication of various optoelectronic devices which necessitate a free form factor on arbitrary substrates. hemispherical imaging system based on a silicon-based photodetector technology combined with interconnected mesh layout supported by elastomeric transfer elements. ${ }^{[5]}$ Yoo et al. reported a fabrication of hydrogenated amorphous silicon (a-Si:H) TFT passive pixel sensor (PPS) array on hemispherical surface using maskless laser-write lithography method. ${ }^{\left[{ }^{[}\right]}$ This successful demonstration of the PPS array on hemispherical surface showed a potential for the fully functional image sensor if it can be fully integrated with the organic photodiodes (OPDs). On the other hand, $\mathrm{Xu}$ et al. demonstrated an OPD Today, most of the current image sensors and cameras are based on flat focal plane array (FPA) technology, including charge coupled devices (CCDs), complementary metal oxide semiconductor (CMOS) imager, or thin film transistors (TFTs) arrays on glass substrates. ${ }^{[1-3]}$ The planar FPA requires complex multielement lenses to correct the image surface; i.e., the Petzval surface. ${ }^{[4]}$ The nonidealities of the optical system can lead to a distorted and blurred image due to off-axis aberrations. To correct these problems, additional optical elements and high precision lens system are required, which result in complex and expensive optical system. Compared to the camera system with the flat FPA, a curved image surface mimicking a spherical human retina provides numerous advantages. ${ }^{[5]}$ The image system with the curved image surface can provide a wide field of view (FOV), a low optical aberration, low $1 / f$ number and less optical lens components leading to compact, cheaper, and lighter camera system. $.4,6,7]$

Over the years, the fabrication of image sensors on curved surface has long been a challenging task among researchers due to the difficulty of developing fabrication methods suitable for curved surfaces. To name a few, Ko et al. developed a

Dr. H. Kim, J. Moon, Prof. J. Kanicki

Department of Electrical Engineering and Computer Science

University of Michigan

Ann Arbor, MI 48109, USA

E-mail: kanicki@umich.edu

Prof. K. Lee

Department of Electrical and Computer Engineering

University of Virginia

Charlottesville, VA 22904, USA

Prof. K. Lee

Department of Material Science and Engineering

University of Virginia

Charlottesville, VA 22904, USA

DOI: $10.1002 / a d m t .201700090$ array on a hemispherical substrate by cold welding method. ${ }^{[9}$ The $11 \times 13$ array of bilayer OPDs based on thermally deposited copper phthalocyanine $(\mathrm{CuPc})$ electron donor and $\mathrm{C}_{60}$ fullerene electron acceptor were fabricated between patterned $\mathrm{Au}$ anode and thin Ag cathode stripes.

So far, very few efforts have been made to use polymer-based OPD materials such as poly(3-hexylthiophene-2,5-diyl): phenyl$\mathrm{C}_{61}$-butyric acid methyl ester (P3HT:PCBM) over a curved surface because of difficulties to deposit solution-based active layers on a nonflat surface. While conventional spin-coating process is inadequate for a curved substrate to produce a uniform thin OPD active layer, alternative deposition options such as spray-coating ${ }^{[10]}$ or ink-jet printing ${ }^{[1-13]}$ could be considered. However, they are still prone to cast a nonuniform OPD layer due to fluidic nature of the solution before forming a solid thin film. Previously, we proposed a novel transfer printing method called double transfer stamping (DTS) process which provides a degree of freedom for on fabrication of polymer based OPDs by enabling solid film transfer from a polydimethylsiloxane (PDMS) stamps to an arbitrary substrate. ${ }^{[14]}$ The elastomeric PDMS stamps for the DTS process can be prepared not only on flat substrate but also on hemispherical surface if an appropriate receiving substrate is fabricated. We previously developed a customized concave glass substrate for the a-Si TFT PPS array fabricated on a hemispherical surface, that we used in this work for hemispherical PDMS stamps development. ${ }^{[8,15]}$ To realize the OPD array on a hemispherical surface, we suggest a novel process combining the modified transfer stamping process and a stereolithography technique to fabricate a 3D mask for metal electrodes deposition over hemispherical surface.

A vision system of primates such as human eyes is regarded as one of the ideal imaging systems due to its wide FOV, low aberration, and low f-number. Its simple one-lens imaging system is enabled by the curved retina, located at the back of the eyeball. ${ }^{[16]}$ Compared to the natural imaging system, the 
artificial imaging system such as CMOS/CCD cameras relies on flat, brittle, silicon-based planar FPAs. Even though both human eyes and artificial cameras resemble in structures, which comprise an aperture, optical elements (lenses), and an FPA, the different shapes of the focal plane distinguish the complexity of overall system, especially in number of lens elements. In modern digital cameras, due to flat shape of the FPA, multiple lens elements with a high precision are required to allow the image surface (Petzval surface) to be flattened so the major optical aberrations such as coma, field curvature, and distortion can be suppressed. ${ }^{[1]}$ This complex lens system became major barrier to develop light-weighted, wide FOV, with free of image distortion imager for next-generation camera systems or artificial eyes. Therefore, it is important to develop novel fabrication methods for a curved imager sensor that can display better quality images using more compact and simpler optics.

A curved image sensor such as retina has an obvious advantage that can reduce the lens system complexity since the edges of the focal plane are located at about the same distance from the lens as from the center. In contrary, the edge of the flat FPA is farther from the lens, while the center is located closer, which results in corner blurriness. ${ }^{[4]}$ To emphasize the advantage of the curved FPA system, we analyzed different FPAs and lens systems using optical modeling. ${ }^{[17]}$

Total three optical systems are considered (Figure 1a-c) and compared. The optical traces for three systems are produced using a ray tracing software, Zemax OpticStudio 16 which is widely used to design camera lens systems. ${ }^{[17]}$ The images are simulated by employing the Image Simulation feature of the software which visualizes geometric aberrations of the modeled optical system which considers lens properties, object radiance, geometric distortion, and spatially variant point spread functions (PSFs) at various wavelengths. The obtained simulation results are shown as image (I)-(III) which are generated from system (a)-(c), respectively. System (a) is Cooke triplet lens system, which is a typical three-lens system used for low-end cameras. ${ }^{[18]}$ System (b) consists of a convex lens $(r=18 \mathrm{~mm})$ and a planar FPA with a $10 \mathrm{~mm}$ diameter. System (c) has same convex lens as System (a) but uses a hemispherical FPA with radius-of-curvature of $r=10 \mathrm{~mm}$. The glass substrate is used as lens material (refractive index $n=1.5168$ ) for all the systems. The excited wavelength for all the systems was $\lambda=546 \mathrm{~nm}$.

As shown in the figure, system (b) with single lens and flat FPA produces an image (II) with the blurred boundaries and barrel distortion. On the other hand, system (a) and (c) can address the problem of system (b) and can generate images with a good quality and with the suppressed geometric aberration. Hence, a curved focal plane of system (c) represents a significant improvement in comparison with the system (a) in terms of simplicity of the lens system. The performance of the optical imaging systems can be characterized by the modulation transfer function (MTF), which is a metric of the optical system ability to transfer contrast from the target object to the image plane at a specific resolution. ${ }^{[19]}$ The obtained MTF for each system is shown in Figure 1(i)-(iii). The different colors of solid lines represent the angles of the tangential incident light rays (black: $0^{\circ}$, green: $13.5^{\circ}$, and blue: $19.1^{\circ}$ ) while the dashed lines represent sagittal incident light rays. We note that system (a) shows inferior MTF performance compared to system (b) and (c); especially the off-axis performance significantly drops to minimum at about 30 cycles $/ \mathrm{mm}$ of the sampling frequency. System (c) shows overall better MTF performance than system (b) while the on-axis performance of system (b) is slightly better than that of system (c) at high sampling frequency. System (c) retained above $50 \%$ of the off-axis modulation up to $19.1^{\circ}$ at 70 cycles $\mathrm{mm}^{-1}$ which corresponds to optical resolution of $7.1 \mu \mathrm{m}$.

From this simple analysis, it is obvious that employing a curved focal plane can significantly reduce the number of lens components required to minimize the optical aberrations, and at the same time we can increase the overall performance of the imaging system.

Despite of many advantages of the hemispherical detector geometry, the realization of this type of curved detector has suffered from extremely challenging fabrication methods compared to well-established process for planar silicon-based FPAs, which comprises conventional photolithographic patterning thin-film deposition, etching, and growth techniques. The fabrication routes for the curved FPA can be largely categorized into two methods. The first one is patterning the circuit on flat substrate, followed by deforming the substrate into desired curved shape. ${ }^{[4,20-22]}$ This method can use the mature semiconductor patterning techniques developed for silicon wafer before the substrate deformation. However, due to the brittle nature of the conventional substrates such as silicon or glass, the strain that can be introduced during the substrate deformation can cause permanent damage and/or modify the devices and circuits performance.

Alternatively, the detector circuits can be directly patterned on the prepared curved substrate by such method as adhesive assisted transfer or cold welding. ${ }^{[5,9,23-25]}$ This method introduces no deformation-induced strain, and allows more freedom in the surface geometry. However, it requires special, unconventional patterning techniques (e.g., radial stretching of PDMS stamp ${ }^{[5]}$ ) to fabricate reliable devices on the curved substrates, which can be potentially quite complicated and difficult to implement. Even though various techniques to fabricate patterned detector arrays on curved surfaces have been reported, very few works have been done to fabricate fully functional detector arrays on the curved substrates. ${ }^{[5]}$ Hence, it is important to investigate new pathways to fabricate photodetector arrays on hemispherical surface.

To realize the FPA array on curved substrate, it is crucial to develop reliable methods to pattern metal electrodes and active layers on the nonflat surface. We propose two novel patterning methods to enable the direct fabrication of metal electrodes and organic active layers on hemispherical substrates. For the electrode deposition, a stereolithgraphy technique used to fabricate the 3D mask is employed. ${ }^{[26-28]}$ The fabricated 3D shadow mask can be conformally attached to the curved surface for the metal electrode patterning process. The organic active layer on the hemispherical surface also requires nonconventional deposition technique other than typical spin-coating process. Here, we adapt modified transfer stamping process previously developed to 3D shaped PDMS stamp.

The 3D shadow mask model was designed based on the dimension information of the hemispherical substrate shown in Figure 2a. The glass substrate is fully customized 
(a)

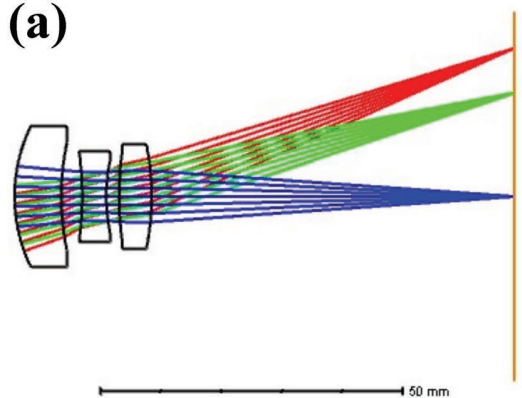

(I)

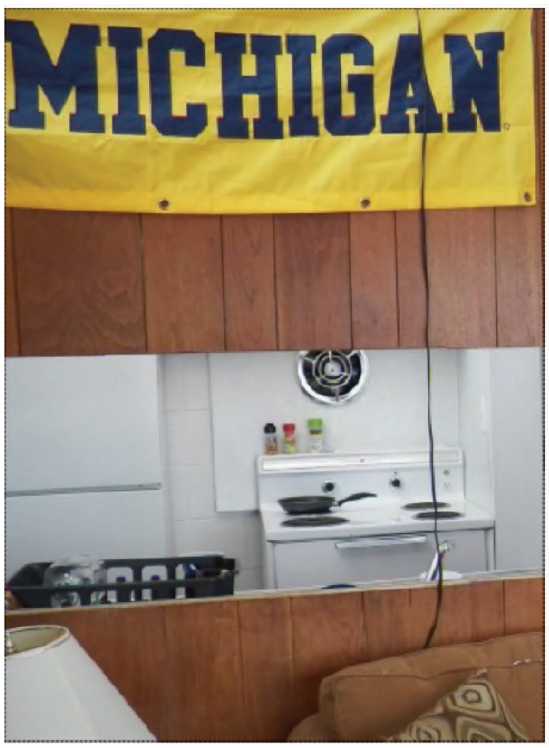

(i)

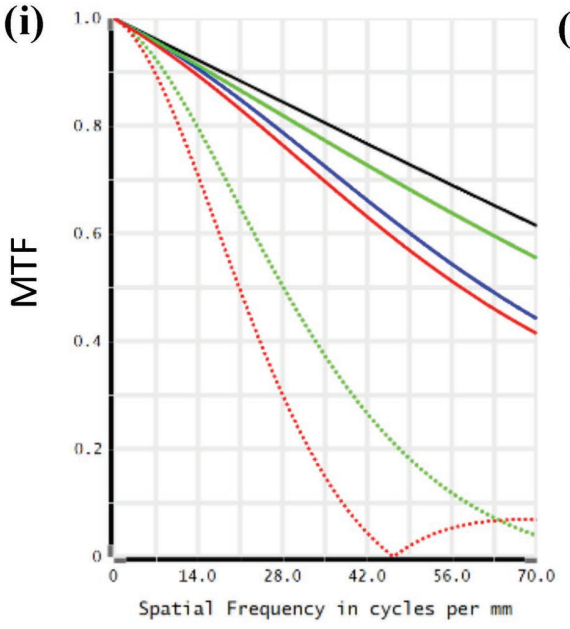

(b)

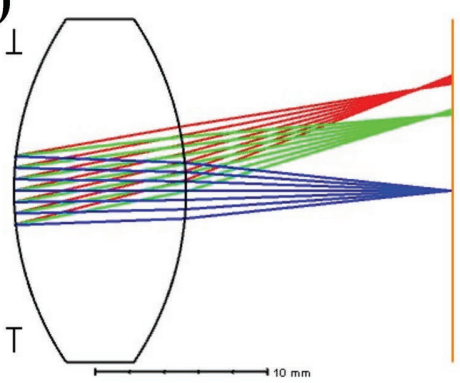

(II)

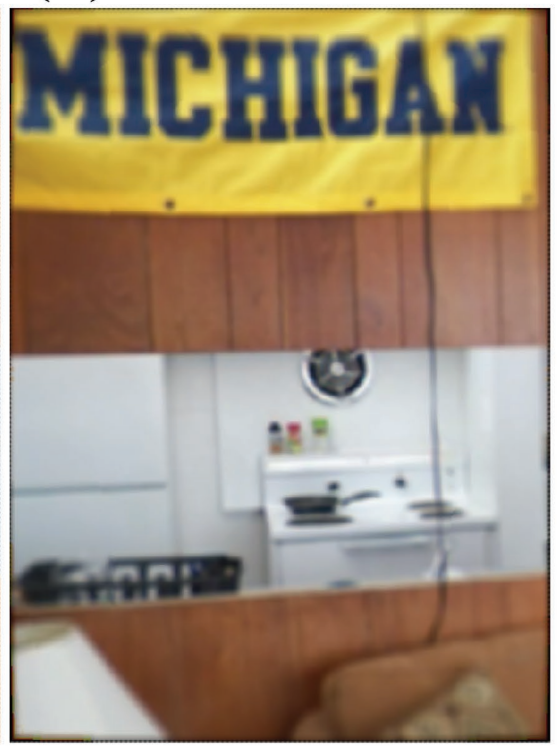

(ii)

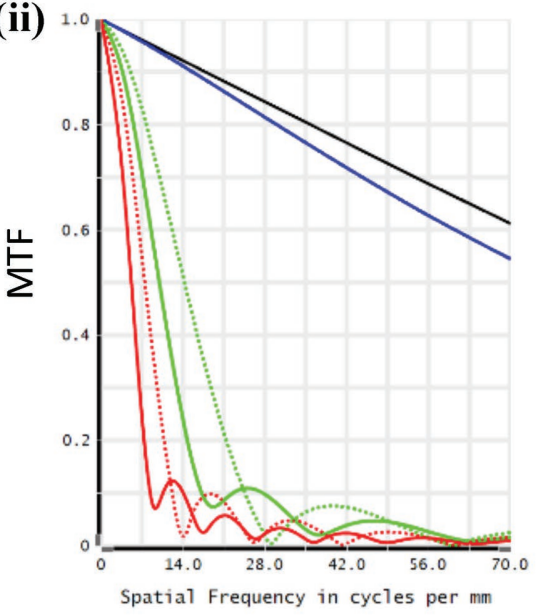

(c)

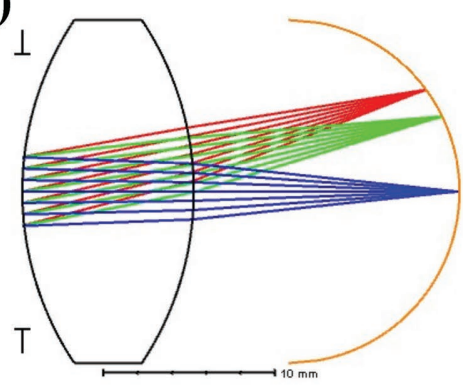

(III)

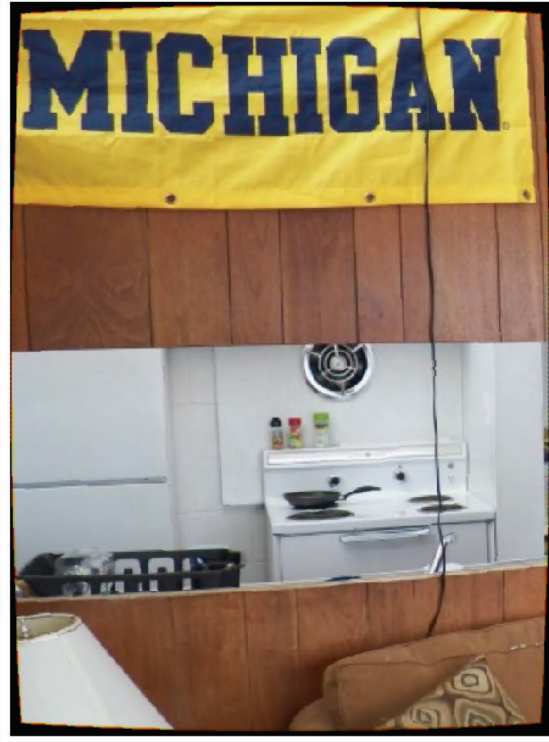

(iii)

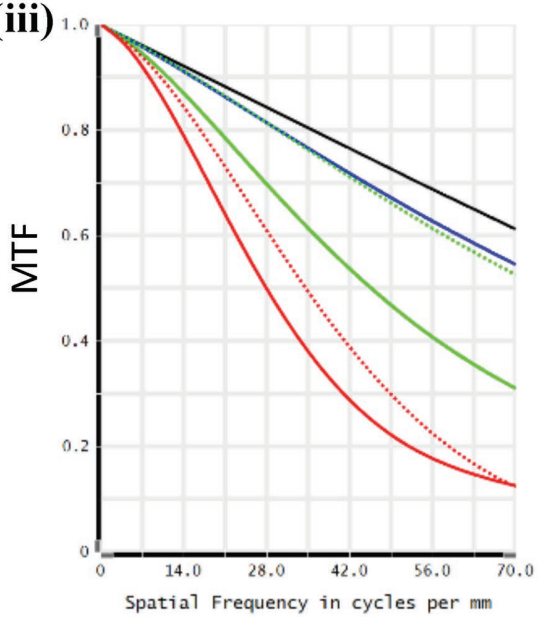

Figure 1. Three different optical systems are shown. a) Cooke triplet, b) flat FPA with single lens, and c) hemipherical FPA ( $r=10 \mathrm{~cm}$ ) with single lens. (I)-(III) Images generated by corresponding optical systems (a-c), respectively. (i)-(iii) MTFs for each system are shown. Black lines corresponds to onaxis $\left(0^{\circ}\right)$, green lines for $13.5^{\circ}$, and blue lines for $19.1^{\circ}$ of incident angles. Both tangential (solid lines) and sagittal image points (dashed lines) are shown.

by a manufacturer to have $\mathrm{FOV}=120^{\circ} .{ }^{[8,15]}$ The prototype $3 \mathrm{D}$ shadow mask were modelled by SolidWorks 2016 (Dassault System, USA) CAD software to have total five uniformly distributed arrays (aperture $=1 \mathrm{~mm}$, pitch $=1 \mathrm{~mm}$ ) to fabricate $5 \times 5$ array. The Solidworks file (.SLDPRT) was converted to .STL format to be compatible with 3D printer's software package. The modeled 3D shadow mask is shown in Figure 3a.

Projet 3500 HDMax (3D Systems, USA) high definition 3D printer (Figure $2 \mathrm{~b}$ ) was used for the 3D shadow mask fabrication with $25 \mu \mathrm{m} x-\gamma$ and $16 \mu \mathrm{m}$ layer thickness deposition 


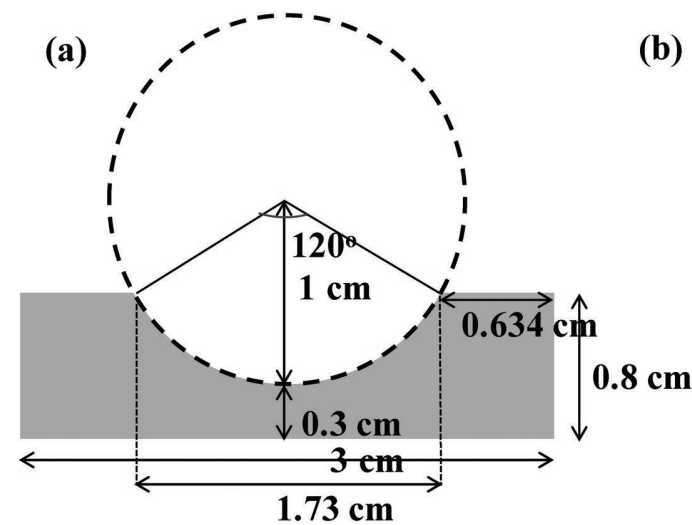

(b)

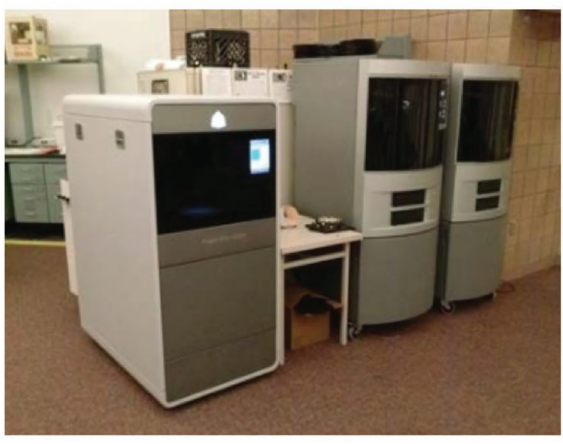

Figure 2. a) Schematic of a hemispherical glass substrate, and b) Projet 3500 HDMax 3D printer used for the fabrication of the 3D shadow mask are shown.

resolution. The imported 3D CAD model of the 3D shadow mask was embedded into the ProJet built-in software with "ultrahigh definition" mode to yield the highest resolution. The Projet 3D printer is based on multijet printing method (MJP method). ${ }^{[29]}$ The MJP process technology employs UV bulbs and photopolymer materials with the piezoprinthead technology to deposit the photocurable plastic resin. The print heads have several piezoelectric nozzle rows arranged in a line. Each layer is exposed to UV for curing for immediate solidification, and the surface is milled to hold tolerance. This process is repeated until fabrication completion. Therefore, they are essentially stereolithography systems..$^{[26,30]}$

For the 3D mask fabrication, VisiJet M3-X (ABS like) resin (3D Systems) with VisiJet S300 Support Wax was used as per manufacturer standard operating protocol. ${ }^{[31]}$ The printed substance required several postprocessing steps to remove the Support Wax to complete the process: (i) oven treatment at $70{ }^{\circ} \mathrm{C}$ for $30 \mathrm{~min}$; (ii) ultrasonic cleaning in a canola oil at $50{ }^{\circ} \mathrm{C} \mathrm{com}$ bined with flushing devices with a warm canola oil $\left(55^{\circ} \mathrm{C}\right)$ for up to $5 \mathrm{~min}$; (iii) washing with Decon 90 detergent for $5 \mathrm{~min}$ to flush out oil and remaining wax.

Figure $3 \mathrm{~b}$ shows a prototype shadow mask fabricated by a $3 \mathrm{D}$ printer. The thickness of the 3D mask was $1 \mathrm{~mm}$ to sustain the shape of the printed model. The thinner 3D printed shadow mask suffered from deformation due to soft material properties. The printed 3D shadow mask is used for both top and bottom electrodes deposition with $90^{\circ}$ vertical overlap to yield total $5 \times 5$ arrays. Finer and more precise pattern can be designed and fabricated for smaller pixel array fabrication. The prototype bottom electrode ( $\mathrm{Au} 100 \mathrm{~nm}$ ) deposited using the fabricated $3 \mathrm{D}$ shadow mask on the hemispherical substrate is shown in Figure 3c.

The elastomeric 3D PDMS stamps were prepared by mixing the Sylgard 184 silicone elastomer (Dow Corning) and a curing agent in clean room (53\% relative humidity, $22{ }^{\circ} \mathrm{C}$ temperature) environment. ${ }^{[32]}$ The two components were mixed homogenerously for $20 \mathrm{~min}$ and degassed in vacuum chamber at the pressure of $\approx 0.4$ psi overnight before being poured onto targeted hemispherical substrate. The concave glass substrate itself is utilized as the mold for the 3D PDMS stamp. The curved glass substrate was placed at the center of a plastic weighing boat and the mixed PDMS solution was poured on the substrate to fully immerse the curved substrate. The PDMS solution was cured at room temperature for 2 days. The cured 3D PDMS stamps were gently peeled off from the substrate then cut into appropriate form for the stamping (Figure 4a). The thickness of the PDMS stamps boundary were typically $\approx 5 \mathrm{~mm}$. The 3D PDMS stamps were attached to backing glass wafers for a conformal transfer process. For the active layer deposition, the modified transfer stamping process was performed by squeezing the active layer solution between a 3D PDMS stamp and the hemispherical substrate. By adapting the modified transfer stamping process, first we successfully demonstrated a uniform deposition of the P3HT:PCBM BHJ film on hemispherical surface. Next, this technique is used for the hemispherical OPD fabrication. Figure $4 \mathrm{~b}$ shows the uniformly
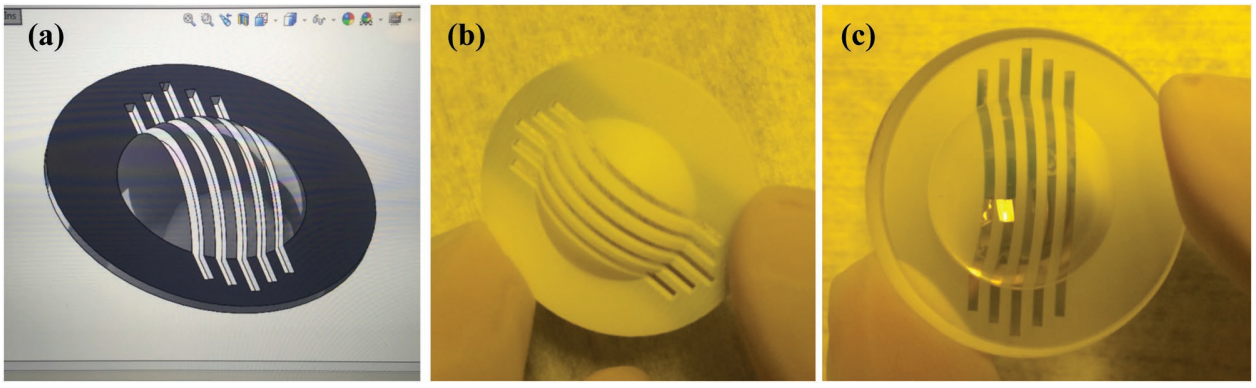

Figure 3. a) Solidworks 3D modeling of the shadow mask, b) 3D printed plastic shadow mask, and c) bottom Au electrode (100 nm) patterned with the $3 \mathrm{D}$ shadow mask on the hemispherical substrate are shown. 


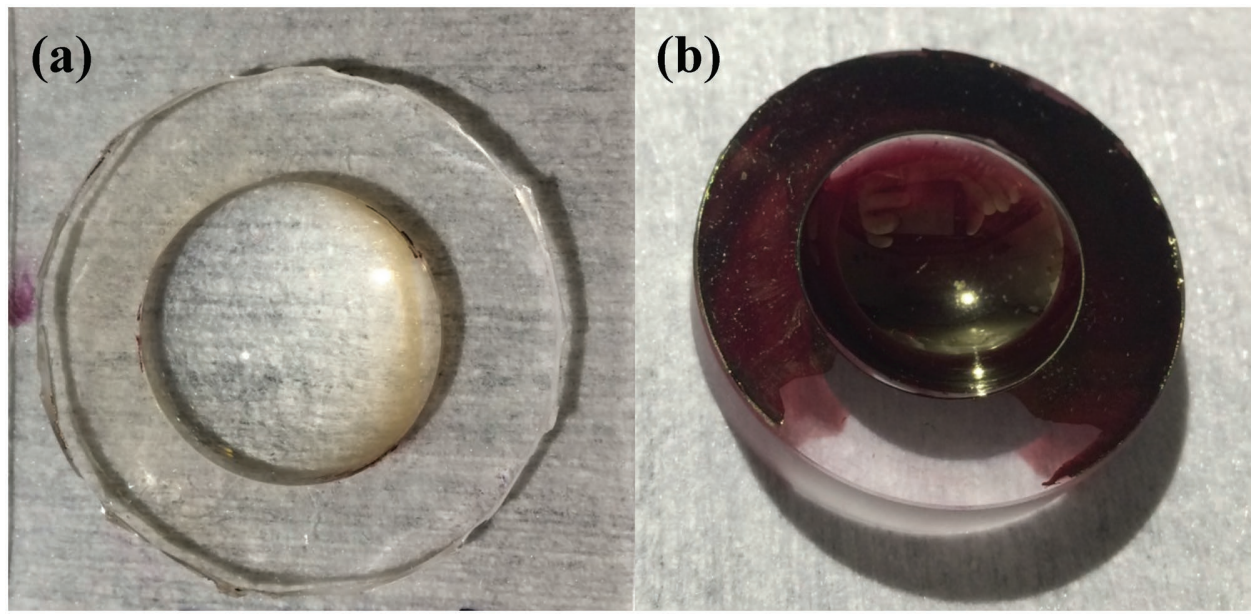

Figure 4. a) Fabricated 3D PDMS stamp for the active layer formation and b) P3HT:PCBM active layer printed on the hemispherical substrate are shown.

printed P3HT:PCBM solution using the fabricated 3D PDMS stamp.

For the fabrication of the hemispherical FPA, we adapt the 3D shadow mask and 3D PDMS stamp described in previous sections. We demonstrate $5 \times 5$ passive pixel array with $1 \mathrm{~mm}^{2}$ feature size, in which the active layer is perpendicularly sandwiched between bottom $\mathrm{Al}$ cathode and top dielectric/metal/ dielectric (DMD) anode stripes. The advantage of DMD structure were described previously. ${ }^{[33]}$ The hemispherical glass substrates were cleaned by acetone, isopropyl alcohol, and deionized water, and treated by $\mathrm{O}_{2}$ plasma $(80 \mathrm{sccm}, 160 \mathrm{mT}$, $300 \mathrm{~W})$ for 1 min subsequently. The prepared 3D shadow mask was attached on the curved substrate and placed inside a thermal evaporator for bottom electrode deposition. The $80 \mathrm{~nm}$ thick aluminum layer was thermally evaporated through the shadow mask with $1 \AA^{-1} \mathrm{~s}^{-1}$ deposition rate (Figure 5a). The PEIE solution was prepared by dissolving $5 \mathrm{wt} \%$ of polyethylenimine ethoxylated (PEIE) (Sigma-Aldrich) in 2-methoxyethanol (2ME, Sigma-Aldrich) solvent in ambient atmosphere. The PEIE solution was spray-coated on top of the Al patterned curved substrate to uniformly deposit $<10 \mathrm{~nm}$ of PEIE dipole layer and annealed at $100{ }^{\circ} \mathrm{C}$ for $10 \mathrm{~min}$ in air. The substrates were transferred into a glove box with nitrogen atmosphere for deposition of photoactive layer. The $20 \mathrm{mg}$ of P3HT (Rieke Metals) and $20 \mathrm{mg}$ of PCBM (American Dye Source, Purity: $>99.5 \%$ ) were mixed into $1 \mathrm{~mL}$ of chlorobenzene and stirred by magnetic bar overnight to produce the $\mathrm{BHJ}$ solution that was filtered by $0.45 \mu \mathrm{m}$ syringe filter subsequently. Next, the BHJ solution was drop-casted on the curved substrate followed by quick squeeze with the 3D shaped PDMS stamp to form a uniform BHJ film on the curved substrate. Once the BHJ film formation is completed the BHJ active layer is thermally annealed with temperature of $150^{\circ} \mathrm{C}$ for $20 \mathrm{~min}$ (Figure $5 \mathrm{~b}$ ). The final thickness of the $\mathrm{BHJ}$ active layer was measured by Dektek profilometer yielding $250 \pm 20 \mathrm{~nm}$. The $\mathrm{BHJ}$ layer formed substrates with the 3D shadow mask were transferred into thermal evaporator to deposit $5 \mathrm{~nm} \mathrm{MoO}, 15 \mathrm{~nm} \mathrm{Ag}$, and $35 \mathrm{~nm} \mathrm{MoO}$ subsequently to form the transparent top anode. In this work, the transparent Ag metal layer needed to be thicker than the optimized DMD electrode ${ }^{[33]}$ to prevent the opens of the metal lines (a)

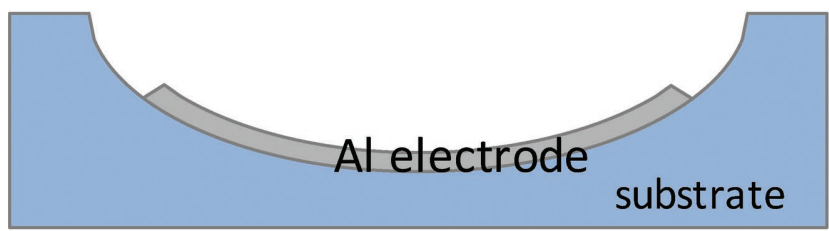

Radius of curvature: $1 \mathrm{~cm}$ Field of View (w/o lens) : $120^{\circ}$

(b)

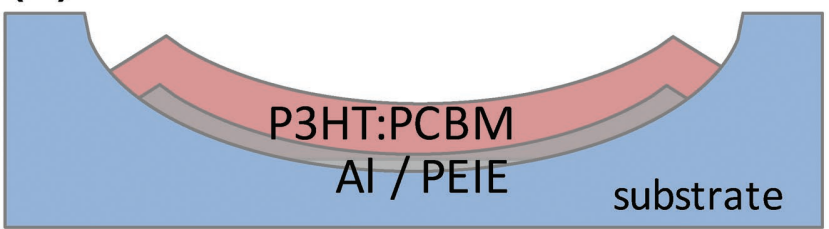

(c)

\section{Encapsulating glass}

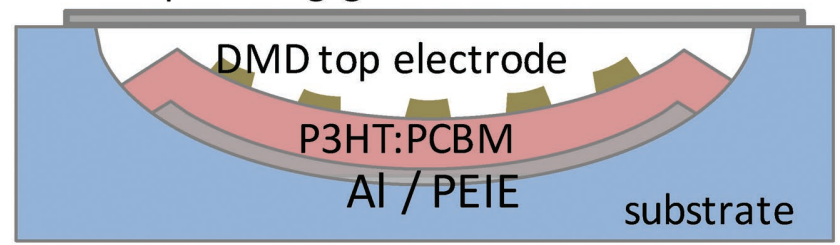

Figure 5. Fabrication procedure of the hemispherical organic photodiode: a) bottom cathode deposition, b) electron transporting dipole layer (PEIE) and $\mathrm{BHJ}$ active layer formation, and c) top DMD anode formation are shown. 


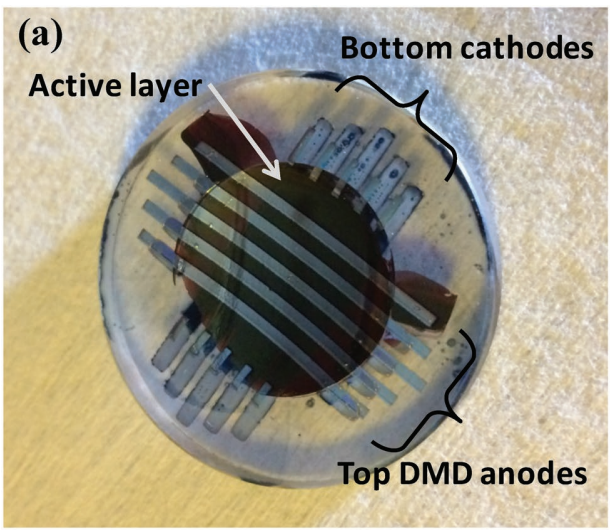

(b)

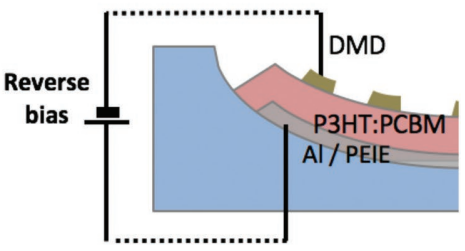

Figure 6. a) Fabricated hemispherical organic photodiode OPD on curved substrate and b) cross-section of the device with polarity applied to electrodes are shown.

over the outside edge of the hemispherical dome. To finish the device fabrication, the devices were encapsulated with a thin slide glass sealed by UV curable epoxy resin to finish the device fabrication (Figure $5 c$ ). An individual square shape device size was $(0.1 \times 0.1) \mathrm{cm}^{2}$ with total of $5 \times 5$ array. The picture of completed hemispherical OPD device is shown in Figure 6a; the bias polarity applied to electrodes is shown in Figure $6 \mathrm{~b}$.

The optoelectronic properties of the fabricated hemispherical OPD were measured and analyzed. One of the most important properties of the OPD is the leakage current under reverse bias condition. The device current density-voltage $(J-V)$ characteristic shown in Figure 7 will allow the determination of such dark leakage current. The irradiance of the illuminated light from the top was $1.63 \mathrm{~mW} \mathrm{~cm}{ }^{-2}$ at $546 \mathrm{~nm}$ wavelength. The $J-V$ characteristics of the hemispherical OPD under illumination and dark conditions were measured by HP2416A semiconductor measurement system with a probe station in a dark

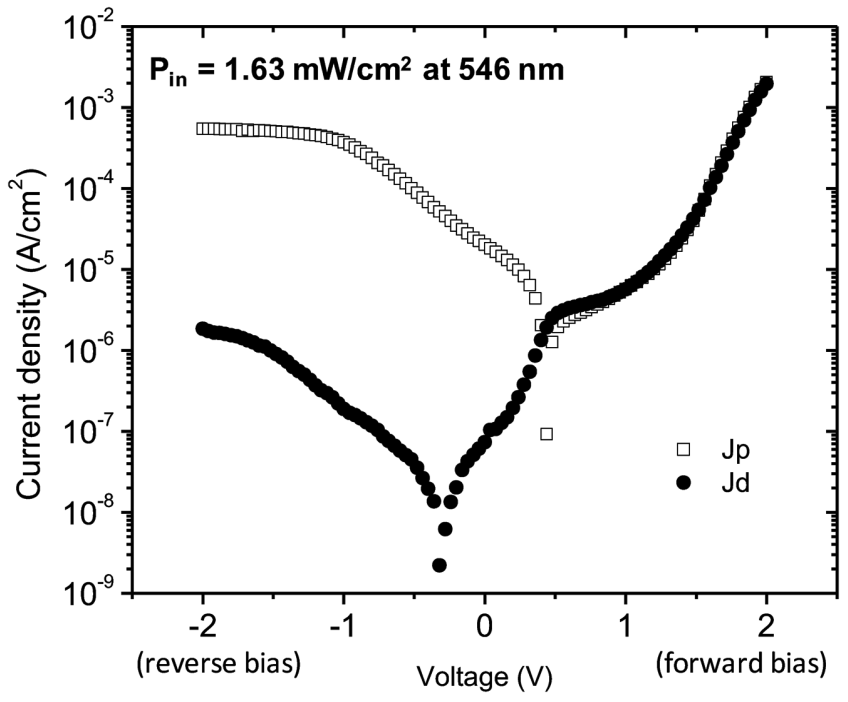

Figure 7. An example of experimental dark and under illumination current density versus voltage characteristics for the hemispherical OPDs are shown. The irradiance of $1.63 \mathrm{~mW} \mathrm{~cm}{ }^{-2}$ light illumination with $546 \mathrm{~nm}$ was used. Solid symbols represent measured dark currents when open symbols are photoresponses.
Faraday cage. The probe contacts were made on the flat periphery region of the electrodes outside the hemispherical dome. The dark current density at $1.5 \mathrm{~V}$ reverse bias of the OPD was $J_{\mathrm{d}}=994 \pm 0.6 \mathrm{nA} \mathrm{cm}{ }^{-2}$, which was a half order lower than previously reported OPD with equivalent active layer thickness. ${ }^{[33]}$ Another important OPD property is the external quantum efficiency (EQE) of the hemispherical OPD that was $17 \% \pm 3 \%$ for $\lambda=546 \mathrm{~nm}$ at $-1.5 \mathrm{~V}$, which is half of the EQE acquired for the $200 \mathrm{~nm}$ DMD OPD fabricated on flat surface. Since the shape of EQE spectrum was similar to one previously reported for the flat surface, ${ }^{[33]}$ it is not shown. This lower EQE can be attributed to thicker Ag electrode (15 nm) used for the top DMD anode that can significantly decrease the optical transmittance of the top transparent anode. To support this claim we used the transfer matrix method to evaluate the light absorption by OPD. Figure 8 shows a simulated contour plot of the light absorption at $546 \mathrm{~nm}$ in the active layer of the OPD as functions of top $\mathrm{MoO}_{3}$ and Ag layer thickness. For this simulation, we used a fixed thickness of P3HT:PCBM = $250 \mathrm{~nm}$ and bottom $\mathrm{MoO}_{3}=5 \mathrm{~nm}$. As it is shown in the figure, while $35 \mathrm{~nm}$ of top $\mathrm{MoO}_{3}$ layer yields maximum optical transmittance, the $15 \mathrm{~nm}$ of Ag layer inevitably decreases absorbance in the active layer down to $43 \%$ which is expected to reduce the EQE performance as experimentally observed. The photodetector performance was evaluated by measuring the specific detectivity $D^{*}=(A \Delta f)^{1 / 2} / \mathrm{NEP}$, where $A$ is the detector area, $\Delta f$ is the bandwidth, and NEP is the noise equivalent power. The NEP can be fairly approximated to NEP $=\left(2 q I_{\mathrm{D}}\right)^{1 / 2} / R$, if we assume that the shot noise current is dominant by dark current over the thermal noise under reverse bias, where $I_{\mathrm{D}}$ is the dark current of the photodetector, $2 q I_{\mathrm{D}}$ is the shot noise power spectral density, and $R$ is responsivity. ${ }^{[34]}$ In this case, we obtain $D^{*}=(1.4 \pm 0.3) \times 10^{11}$ Jones for a $1 \mathrm{~Hz}$ bandwidth at $546 \mathrm{~nm}$

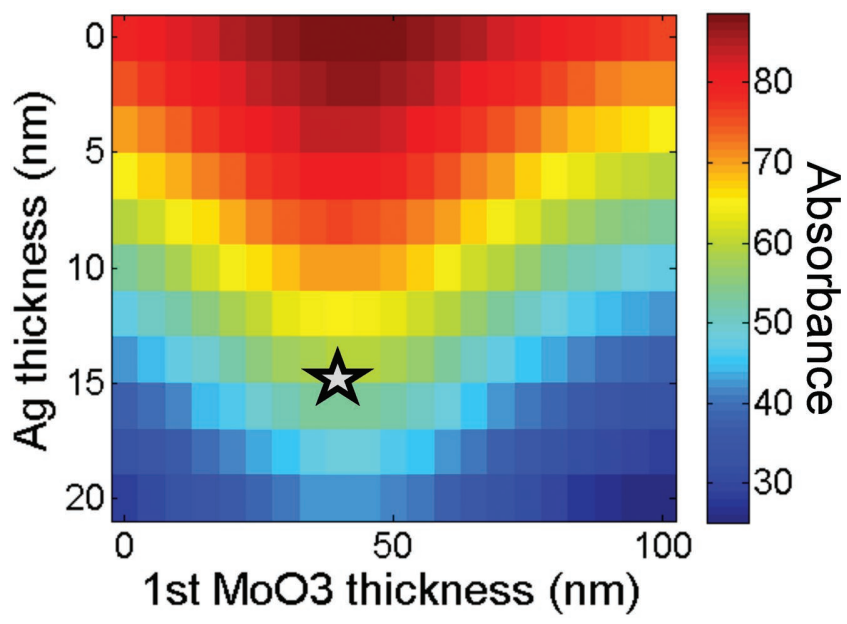

Figure 8. Simulated 2D absorption spectrum with varied top $\mathrm{MoO}_{3}$ and $\mathrm{Ag}$ layer thickness. The color bar on the right side represents the absorbance in P3HT:PCBM at $546 \mathrm{~nm}$ while the star indicate the chosen thickness in this work. 
wavelength for $-1.5 \mathrm{~V}$ bias. The $D *$ value obtained here is comparable with the $D^{*}$ of the $200 \mathrm{~nm}$ DMD OPD device fabricated on flat surface. ${ }^{[33]}$

The fabricated hemispherical OPDs showed reduced aging effect after storing for more than one month in air. This is more likely due to inverted top-anode OPD structure and appropriate encapsulation process used in this study. Since the inverted organic solar cell structures showed superior stability in air compared to conventional structures, ${ }^{[35-37]}$ we anticipate the lifetime of our devices can be comparable with its inorganic counterparts after proper encapsulation. Finally, we found that the fabrication yield of the hemispherical OPD array under described laboratory conditions was $\approx 10 \%$ for sample number $N>50$. The low yield is possibly due to nonuniform nature of the DMD transparent top anode fabricated using vertical thermal evaporation process; this is especially true for the transition region between hemispherical surface and flat suface of the substrate periphery. A modification of the vacuum evaporator is needed for more uniform thin metal evaporation process that could result in yield improvements through reduction of the open lines.

In conclusion, for the first time, we demonstrated a novel method to fabricate organic photodiode passive arrays on a hemispherical substrate with FOV $=120^{\circ}$. To realize the hemispherical OPD fabrication, two unconventional fabrication processes were developed: 3D shadow mask fabrication by sterolithgraphy, and 3D PDMS stamp for transfer printing on curved substrate. These techniques allow direct patterning of the device electrodes without an excessive strain caused by substrate deformation process. We believe that the developed 3D mask and 3D organic layer transfer printing process can be extended to active-matrix arrays allowing the fabrication of more complex structure leading to imaging system such as artificial human eyes.

\section{Acknowledgements}

The authors would like to thank Shawn O'Grady in the University of Michigan 3D Lab for his support and consultation on the 3D printing of the shadow masks.

\section{Conflict of Interest}

The authors declare no conflict of interest.

\section{Keywords}

curved, hemispherical, stereolithography

Received: April 10, 2017

Revised: May 20, 2017

Published online: July 18, 2017

[1] T. S. L. Gerald, C. Holst, CMOS/CCD Sensors and Camera Systems, JCD Publishing, FL, USA 2007.

[2] J. Ohta, Smart CMOS Image Sensors and Applications, CRC Press, FL, USA, 2008.
[3] C. Zhao, J. Kanicki, Med. Phys. 2014, 41, 91902.

[4] S.-B. Rim, P. B. Catrysse, R. Dinyari, K. Huang, P. Peumans, Opt. Express 2008, 16, 4965.

[5] H. C. Ko, M. P. Stoykovich, J. Song, V. Malyarchuk, W. M. Choi, C.-J. Yu, J. B. GeddesIII, J. Xiao, S. Wang, Y. Huang, J. A. Rogers, Nature 2008, 454, 748.

[6] L. P. Lee, L. P. Lee, R. Szema, Science 2010, 1148, 1148.

[7] H.-C. Jin, J. R. Abelson, M. K. Erhardt, R. G. Nuzzo, J. Vac. Sci. Technol., B: Microelectron. Nanom. Struct. -Process., Meas., Phenom. 2004, 22, 2548

[8] G. Yoo, T. Fung, D. Radtke, M. Stumpf, U. Zeitner, J. Kanicki, Sens. Actuators, A 2010, 158, 280.

[9] X. Xu, M. Davanco, X. Qi, S. R. Forrest, Org. Electron. 2008, 9, 1122.

[10] S. F. Tedde, J. Kern, T. Sterzl, J. Fu, P. Lugli, O. Hayden, Nano Lett. 2009, 9, 980 .

[11] S. Lilliu, M. Böberl, M. Sramek, S. F. Tedde, J. E. MacDonald, O. Hayden, Thin Solid Films 2011, 520, 610.

[12] H. Gorter, M. J. J. Coenen, M. W. L. Slaats, M. Ren, W. Lu, C. J. Kuijpers, W. A. Groen, Thin Solid Films 2013, 532, 11.

[13] A. Teichler, J. Perelaer, U. S. Schubert, J. Mater. Chem. C 2013, 1, 1910.

[14] H. Kim, B. Song, K. Lee, S. Forrest, J. Kanicki, Adv. Opt. Mater. 2017, 5, 1600784

[15] G. Yoo, H. Lee, D. Radtke, M. Stumpf, U. Zeitner, J. Kanicki, Microelectron. Eng. 2010, 87, 83.

[16] E. Allen, S. Triantaphillidou, S. Triantaphillidou, The Manual of Photography and Digital Imaging, 10th ed., CRC Press, FL, USA, 2011.

[17] J. M. Geary, Introduction to Lens Design: With Practical ZEMAX Examples, Willmann-Bell Richmond, VA, USA, 2002.

[18] M. J. Kidger, Fundamental Optical Design, SPIE Press, WA, USA 2001.

[19] G. D. Boreman, Modulation Transfer Function in Optical and ElectroOptical Systems, SPIE Press, WA, USA, 2001.

[20] K. Itonaga, T. Arimura, K. Matsumoto, G. Kondo, K. Terahata, S. Makimoto, M. Baba, Y. Honda, S. Bori, T. Kai, K. Kasahara, M. Nagano, M. Kimura, Y. Kinoshita, E. Kishida, T. Baba, S. Baba, Y. Nomura, N. Tanabe, N. Kimizuka, Y. Matoba, T. Takachi, E. Takagi, T. Haruta, N. Ikebe, K. Matsuda, T. Niimi, T. Ezaki, T. Hirayama, IEEE Symp. on VLSI Technology (VLSI-Technology): Digest of Technical Papers, IEEE, 2014, pp. 1-2.

[21] R. Dinyari, J. D. Loudin, P. Huie, D. Palanker, P. Peumans, Tech Dig. - Int. Electron Devices Meet. 2009, 595 pp. 1-4.

[22] D. Dumas, M. Fendler, N. Baier, J. Primot, E. le Coarer, Appl. Opt. 2012, 51, 5419

[23] S. R. Forrest, X. Xu, C. K. Renshaw, M. Hack, J. Brown, 23rd Annual Meeting of the IEEE Photonic Society, IEEE, 2010, p. 369-370.

[24] X. Xu, M. Mihnev, A. Taylor, S. R. Forrest, Appl. Phys. Lett. 2009, 94, 9.

[25] T. F. O'Connor, A. V. Zaretski, B. A. Shiravi, S. Savagatrup, A. D. Printz, M. I. Diaz, D. J. Lipomi, Energy Environ. Sci. 2014, 7, 370.

[26] M. Vaezi, H. Seitz, S. Yang, Int. J. Adv. Manuf. Technol. 2013, 67, 1721.

[27] D. I. Wimpenny, P. M. Pandey, L. J. Kumar, Advances in 3D Printing $\&$ Additive Manufacturing Technologies, Springer Verlag, Singapor, 2017.

[28] T. J. Snyder, M. Andrews, M. Weislogel, P. Moeck, J. Stone-Sundberg, D. Birkes, M. P. Hoffert, A. Lindeman, J. Morrill, O. Fercak, S. Friedman, J. Gunderson, A. Ha, J. McCollister, Y. K. Chen, J. Geile, A. Wollman, B. Attari, N. Botnen, V. Vuppuluri, J. Shim, W. Kaminsky, D. Adams, J. Graft, 3D Printing and Additive Manufacturing 2014, 1(3), 169-176.

[29] G. Andreas, J. Hotter, Additive Manufacturing: 3D Printing for Prototyping and Manufacturing, Carl Hanser Verlag GMBH Co KG, Munich, Germany 2013. 
[30] K. Ikuta, S. Maruo, S. Kojima, Proc. MEMS 98. Eleventh Annual International Workshop, IEEE, 1998, p. 290.

[31] 3D Systems, Projet ${ }^{\circledR} 3500$ SD \& HD Professional 3D Printers, 2014.

[32] Y. Xia, G. M. Whitesides, Annu. Rev. Mater. Sci. 1998, 28, 153.

[33] H. Kim, K.-T. Lee, C. Zhao, L. J. Guo, J. Kanicki, Org. Electron. 2015, 20, 103.
[34] J.-M. Liu, Photonic Devices, Cambridge University Press, NY, USA, 2005.

[35] E. Saracco, B. Bouthinon, J. Verilhac, C. Celle, N. Chevalier, D. Mariolle, O. Dhez, J. Simonato, Adv. Mater. 2013, 25, 6534.

[36] B. Zimmermann, U. Würfel, M. Niggemann, Sol. Energy Mater. Sol. Cells 2009, 93, 491.

[37] B. A. E. Courtright, S. A. Jenekhe, ACS Appl. Mater. Interfaces 2015, 7, 26167. 